\title{
CTLA-4 gene polymorphisms and their influence on predisposition to autoimmune thyroid diseases (Graves' disease and Hashimoto's thyroiditis)
}

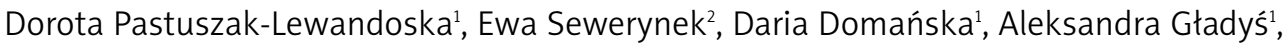 \\ Renata Skrzypczak ${ }^{1}$, Ewa Brzeziańska ${ }^{1}$
}

1Department of Molecular Bases of Medicine, $1^{\text {st }}$ Chair of Internal Diseases, Medical University of Lodz, Poland

2Department of Endocrine Disorders and Bone Metabolism, 1'st Chair of Endocrinology, Medical University of Lodz, Poland

Submitted: 20 January 2011

Accepted: 16 April 2011

Arch Med Sci 2012; 8, 3: 415-421

DOI: 10.5114 /aoms.2012.28593

Copyright (c) 2012 Termedia \& Banach
Corresponding author: Ewa Brzeziańska MSc, PhD Department of Molecular Bases of Medicine 1st Chair of Internal Diseases Medical University of Lodz 251 Pomorska 92-213 Lodz, Poland Phone: +48 426757715 E-mail: ewa.brzezianska@umed.lodz.pl

\section{Abstract}

Introduction: Autoimmune thyroid disease (AITD) is associated with both genetic and environmental factors which lead to the overactivity of immune system. Cytotoxic T-Lymphocyte Antigen 4 (CTLA-4) gene polymorphisms belong to the main genetic factors determining the susceptibility to AITD (Hashimoto's thyroiditis, HT and Graves' disease, GD) development. The aim of the study was to evaluate the relationship between CTLA-4 polymorphisms (A49G, $1822 \mathrm{C} / \mathrm{T}$ and $\mathrm{CT} 60 \mathrm{~A} / \mathrm{G}$ ) and $\mathrm{HT}$ and/or GD in Polish patients.

Material and methods: Molecular analysis involved AITD group, consisting of HT $(n=28)$ and GD $(n=14)$ patients, and a control group of healthy persons $(n=20)$. Genomic DNA was isolated from peripheral blood and CTLA-4 polymorphisms were assessed by polymerase chain reaction-restriction fragment length polymorphism method, using three restriction enzymes: Fnu4HI (A49G), BsmAl (1822 C/T) and BsaAl (CT60 A/G).

Results: Statistical analysis ( $\chi^{2}$ test) confirmed significant differences between the studied groups concerning CTLA-4 A49G genotypes. CTLA-4 A/G genotype was significantly more frequent in AITD group and OR analysis suggested that it might increase the susceptibility to HT. In GD patients, OR analysis revealed statistically significant relationship with the presence of $G$ allele. In controls, CTLA-4 A/A genotype frequency was significantly increased suggesting a protective effect. There were no statistically significant differences regarding frequencies of other genotypes and polymorphic alleles of the CTLA-4 gene (1822 C/T and $\mathrm{CT} 60 \mathrm{~A} / \mathrm{G}$ ) between the studied groups.

Conclusions: CTLA-4 A49G polymorphism seems to be an important genetic determinant of the risk of HT and GD in Polish patients.

Key words: genetic factors, autoimmune thyroid disease, single nucleotide polymorphism, allelic polymorphism.

\section{Introduction}

Autoimmune thyroid disease (AITD) is the most prevalent autoimmune disorder, related to cellular and humoral immune responses targeted at the thyroid gland and affecting up to $5 \%$ of the general population [1]. According to the most recent epidemiological studies on different populations, Graves' disease (GD) and Hashimoto thyroiditis (HT) are recog- 
nized as the most common forms of AITD. These types of autoimmune thyroid disorder display a multifactorial pattern of inheritance, with both genetic and environmental factors influencing their development [2-5]. The major identified AITD susceptibility genes are classified in two functional groups: 1) immune regulatory genes: cytotoxic $T$ lymphocyte-associated antigen 4 (CTLA-4); protein tyrosine phosphatase, nonreceptor type 22 (PTPN22); interleukin 2 receptor (IL-2R); and 2) thyroid-specific genes: the thyroglobulin gene $(T G)$ and the thyrotropin receptor gene (TSHR) [2, 6-8].

Human CTLA-4 is one of the most thoroughly studied loci (2q33) concerning autoimmunity and relevance in GD and/or HT development. The CTLA-4 encodes an immunoregulatory molecule, expressed on the surface of activated T cells, and via interaction with the B7 molecule downregulates T-cell activation [9]. It is claimed that T-effector activity could be determined by CTLA-4 single nucleotide polymorphisms (SNPs) [10]. Especially, A49G dimorphism (Thr/Ala exchange in a peptide) leads to the expression of defective receptor, and, as a result, the inhibitory effect of CTLA-4 on lymphocyte T cell activation is impaired [11, 12].

The association between AITD (HT and/or GD) and CTLA-4 polymorphisms (A49G, $1822 \mathrm{C} / \mathrm{T}$ and CT60 A/G and some other polymorphic sites) has been confirmed in several studies [13-17]. Particularly, CTLA-4 A49G and CT60 polymorphisms have been correlated with susceptibility to AITD development $[18,19]$.

The aim of the present study was to assess the frequency of selected CTLA-4 polymorphisms (A49G, 1822 C/T and CT60 A/G) in a group of Polish patients with diagnosed $\mathrm{GD}$ and $\mathrm{HT}$ and to evaluate the association between CTLA-4 genotypes and susceptibility to AITD (HT and/or GD) development - particularly as it has not been studied thoroughly so far.

\section{Material and methods}

The study was approved by the Committee for Bioethics of the Medical University of Lodz, Poland.

\section{Biological material}

Blood samples (200 $\mu \mathrm{l}$, EDTA-collected) were obtained from 42 patients who were diagnosed and treated in the Outpatient Clinic at the Regional Centre of Menopause and Osteoporosis in the Military Hospital of the Medical University of Lodz. The HT group consisted of 28 patients ( 27 women and 1 man, aged 21-81 years, median: 49 years). The GD group included 14 patients ( 10 women and 4 men, aged $25-72$ years, median: 37 years). The control group consisted of 20 healthy persons ( 15 women, 5 men, aged $23-55$ years, median: 36 years).

\section{Patients' clinical characteristics}

Patients were diagnosed based on clinical symptoms and signs, and the results of biochemical analysis performed using enhanced chemiluminescence (ECL) assays (Boehringer Mannheim, Germany): Elecsys Anti-Tg test (human antigens and human monoclonal antibodies against thyroglobulin, Tg-Ab), Elecsys Anti-TPO test (recombinant antigens and human polyclonal antibodies against thyroid peroxidase, TPO-Ab), Elecsys Anti-TSHR test (human monoclonal antibodies against thyrotropin receptor, TSHR-Ab).

The diagnosis of GD was based on the presence of an elevated level of TSHR-Ab (> $1.5 \mathrm{U} / \mathrm{l})$. In this group the majority of patients had hyperthyroidism with diffuse goiter, decreased value of thyroid-stimulating hormone $(\mathrm{TSH})(<0.27 \mathrm{ulU} / \mathrm{ml})$, and high levels of free thyroid hormones (fT3 $>4.4 \mathrm{pg} / \mathrm{ml}$, fT4 $>1.7 \mathrm{ng} / \mathrm{ml}$ ). There were also patients with subclinical hyperthyroidism with low levels of TSH and thyroid hormones in the normal range. Additionally, three GD patients (3/14), including 2 women and 1 man, had Graves' orbitopathy with levels of TSHR-Ab higher than $6 \mathrm{IU} / \mathrm{l}$.

The HT was recognized when elevated levels of anti-TPO were observed (> $34 \mathrm{lU} / \mathrm{ml}$ ). In this group there were patients with hypothyroidism when an elevated level of serum TSH $(>5.0 \mathrm{ulU} / \mathrm{ml})$ and low levels of fT3 and fT4 hormones $(<0.93 \mathrm{pg} / \mathrm{ml}$ and $<2.0 \mathrm{pg} / \mathrm{ml}$ respectively) were observed and subclinical hypothyroidism with TSH in the range $5-10 \mathrm{ulU} / \mathrm{ml}$ and thyroid hormones in the normal range.

The HT and GD patients were treated according to the recommended treatment standards. The control group consisted of healthy persons with no family history of thyroid disorders and/or autoimmune diseases, biochemically and clinically euthyroid.

\section{DNA isolation}

Genomic DNA was extracted using QIAamp DNA Mini Kit (Qiagen, Germany), according to the manufacturer's protocol. Quality and quantity of each DNA sample were assessed spectrophotometrically (NanoDrop Spectrophotometer ND-1000, ThermoScientific, USA).

\section{Analysis of CTLA-4 polymorphism}

CTLA-4 polymorphisms (A49G, 1822 C/T and $\mathrm{CT} 60 \mathrm{~A} / \mathrm{G}$ ) were assessed by PCR-RFLP (polymerase chain reaction-restriction fragment length polymorphism), using the following restriction enzymes: Fnu4HI, BsmAl, BsaAl (New England Biolabs, USA).

First, genomic DNA sequences containing the polymorphic region, i.e., Fnu4HI or BsmAl or BsaAl restriction site, were amplified in a PCR reaction 
Table I. The sequences of primers (forward and revere) for the studied polymorphic CTLA-4 variants

\begin{tabular}{|lll|}
\hline Polymorphism & Forward primer $\left(5^{\prime} \rightarrow 3^{\prime}\right)$ & Reverse primer $\left(5^{\prime} \rightarrow 3^{\prime}\right)$ \\
\hline CTLA-4 A49G & -GCTCAGCTGAACCTGGCT- & -AAATCACTGCCCTTGACTGC- \\
\hline CTLA-4 1822 C/T & -TCAAAGGGATTGAGCAGATG- & -TCCCATGCTCCTTTGTTCTC- \\
\hline CTLA-4 CT60 A/G & -GATTTCTTCACCACTATTTGG GATATTAC- & -AGATCAAAATGGCTGCAAGG- \\
\hline
\end{tabular}

(Personal Thermocycler, Eppendorf, Germany), in a total volume of $25 \mu \mathrm{l}$, including: $3 \mu \mathrm{l} 10 \mathrm{x}$ AmpliTaq Gold ${ }^{\oplus} 360$ buffer (150 mM Tris-HCl, pH 8.3, 500 mM KCl), $0.12 \mu \mathrm{l}(5 \mathrm{U} / \mu \mathrm{l})$ AmpliTaq Gold ${ }^{\oplus} 360$ DNA Polymerase, $2 \mu \mathrm{l}(25 \mathrm{mM}) \mathrm{MgCl}_{2}, 0.66 \mu \mathrm{l}(10 \mathrm{mM})$ dNTPs (Applied Biosystems, USA), $1 \mu \mathrm{l}$ (40 ng) DNA, $2.4 \mu \mathrm{l}(1.2 \mu \mathrm{l} 0.5 \mu \mathrm{M}$ each primer: forward and reverse) and $15.82 \mu \mathrm{l}$ nuclease-free water. The same annealing temperature of $58^{\circ} \mathrm{C}$ was applied for each studied polymorphism. The amplification reaction was performed for 29 cycles. As a control for PCR contamination, blank samples were used with nuclease-free water instead of DNA. Primer sequences are presented in Table I.

The PCR products of the studied CTLA-4 polymorphisms were genotyped based on SNPs reported as associated with AITD susceptibility (marker IDs according to dbSNP: rs231775 for CTLA-4 A49G, rs231779 for CTLA-4 $1822 \mathrm{C} / \mathrm{T}$ and rs3087243 for CTLA-4 CT60 A/G) [17, 20]. Genotyping was carried out by digesting the amplified PCR products with the restriction enzymes, Fnu4HI recognizing CTLA-4 A49G, BsmAl recognizing CTLA-4 1822 C/T and BsaAl recognizing CTLA-4 CT60 A/G, as follows: $10 \mu \mathrm{ICCR}$ product was incubated at $37^{\circ} \mathrm{C}$ for $6 \mathrm{~h}$ with $1 \mathrm{U}$ of a given enzyme in a total volume of $20 \mu \mathrm{l}$, according to manufacturer's protocol. The digestion procedure was repeated three times.

The PCR-RFLP products $(10 \mu \mathrm{l})$ were separated in $2 \%$ agarose gel at $5 \mathrm{~V} / \mathrm{cm}$ for $2 \mathrm{~h}$ in $1 \mathrm{x}$ TBE buffer (Tris/Borate/EDTA) and then assessed in UV light (InGenius Bio Imaging analysis system, Syngene, UK). The lengths of PCR-RFLP products were evaluated using DNA pUC19/Mspl length marker (DNA Gdansk, Poland), and were as follows: 1) CTLA-4 A49G: 84 bp for A/A, 106 bp for G/G and both products for A/G genotype; 2) CTLA-4 1822 C/T: 102 bp for $\mathrm{T} / \mathrm{T}, 74 \mathrm{bp}$ for $\mathrm{C} / \mathrm{C}$ and both products for $\mathrm{T} / \mathrm{C}$ genotype; 3) CTLA-4 CT60 A/G: 102 bp for A/A, 68 bp for $\mathrm{G} / \mathrm{G}$ and both products for $A / G$ genotype.

\section{Statistical analysis}

Allele and genotype frequencies were compared between HT, GD and control groups using the $\chi^{2}$ test. Values of $p$ below $0.05(p<0.05)$ were considered as statistically significant. Odd ratios (OR) and their $95 \%$ confidence intervals $(95 \% \mathrm{Cl})$ were calculated as an assessment of strength and direction of association between individual genotype and susceptibility to AITD (HD and/or GD) development. The relative risk (assessed as OR) defines the level of risk for AITD patients (HT and/or GD) compared to the control group. Hardy-Weinberg equilibrium was assessed using the $\chi^{2}$ test to compare the genotypes.

For statistical analysis the Med-Calc 11.4.3.0 statistical software for Windows was used.

\section{Results}

The PCR products of the studied CTLA-4 polymorphisms were genotyped based on SNPS. An example of genotype analysis using the RFLP-PCR method is shown in Figure 1.

Statistical analysis ( $\chi^{2}$ test) confirmed the presence of significant differences between the studied groups concerning the distribution of CTLA-4 A49G genotypes. CTLA-4 A/G genotype was significantly more frequent in the AITD group (0.60 AITD vs. 0.25 control, $\mathrm{OR}=4.40,95 \% \mathrm{Cl}: 1.35-14.43$, $p=0.01$ ) and A/A genotype frequency was increased in the control group (0.10 AITD vs. 0.50 control group, $\mathrm{OR}=0.11,95 \% \mathrm{Cl}: 0.03-0.41, p=0.001)$.

Following the statistical tendency, the frequency of CTLA-4 A49G genotypes in HT and GD patients in comparison with controls was analyzed.

The results showed significantly increased $A / G$ genotype frequency in HT patients in comparison with controls $(p<0.05)$. The frequency of A/A genotype was significantly higher in the control group $(p<0.05)$. There were no significant differences in distribution of $A$ and $G$ alleles between the studied groups $(p>0.05)$. Statistical analysis results are shown in Table II.

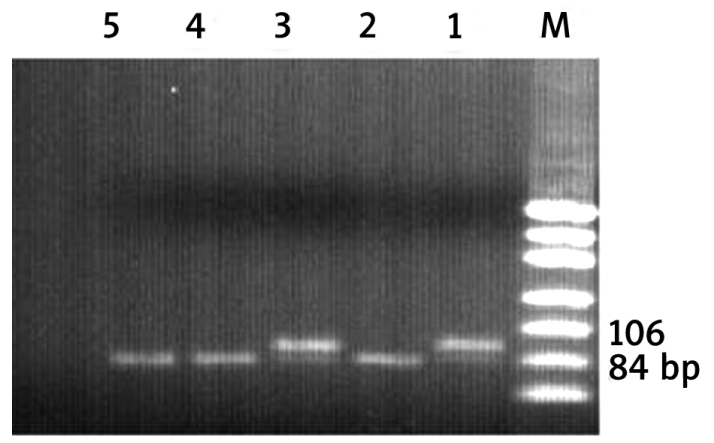

Figure 1. Electropherogram of PCR-RFLP products of CTLA-4 A49G genotypes; Fnu4HI digestion (A/G polymorphism)

Lines 1, 3 - heterozygous $A / G$ patients, with $G D$ and $H T$, respectively, lines 2, 4, 5 - homozygous $A / A$ patients with GD, M-DNA pUC19/Mspl length marker 
Table II. The frequencies of CTLA4 A49G genotypes (A/A, A/G, G/G) as well as A and $G$ alleles in HT patients in comparison with control group

\begin{tabular}{|c|c|c|c|c|c|c|}
\hline \multirow{2}{*}{$\begin{array}{l}\text { CTLA-4 } \\
\text { A49G } \\
\text { genotype }\end{array}$} & \multicolumn{2}{|c|}{$\mathrm{HT}(N=28)$} & \multicolumn{2}{|c|}{ Control group $(N=20)$} & \multirow{2}{*}{$\begin{array}{l}\text { Odd ratio } \\
(95 \% \mathrm{Cl})\end{array}$} & \multirow[t]{2}{*}{ Value of $p$} \\
\hline & $n$ & Frequency & $n$ & Frequency & & \\
\hline $\mathrm{A} / \mathrm{A}$ & 3 & 0.11 & 10 & 0.50 & $0.12(0.03-0.53)$ & 0.0051 \\
\hline$A / G$ & 19 & 0.68 & 5 & 0.25 & $6.33(1.75-22.91)$ & 0.0049 \\
\hline $\mathrm{G} / \mathrm{G}$ & 6 & 0.21 & 5 & 0.25 & $0.55(0.13-2.22)$ & 0.7700 \\
\hline A allele & 25 & 0.45 & 25 & 0.63 & $0.48(0.21-1.11)$ & 0.0900 \\
\hline G allele & 31 & 0.55 & 15 & 0.38 & $1.24(0.51-3.02)$ & 0.0901 \\
\hline
\end{tabular}

Table III. The frequencies of CTLA4 A49G genotypes (A/A, A/G, G/G) as well as A and G alleles in GD patients in comparison with control group

\begin{tabular}{|lccccccc|}
\hline $\begin{array}{l}\text { CTLA-4 } \\
\text { A49G } \\
\text { genotype }\end{array}$ & \multicolumn{2}{c}{ GD $(N=14)$} & Control group $(N=20)$ & & $\begin{array}{c}\text { Odd ratio } \\
(95 \% \mathrm{Cl})\end{array}$ & Value of $p$ \\
\cline { 2 - 6 } & $n$ & Frequency & $n$ & Frequency & & $0.08(0.01-0.71)$ & 0.02 \\
\hline A/A & 1 & 0.07 & 10 & 0.50 & $2.25(0.52-9.73)$ & 0.28 \\
\hline A/G & 6 & 0.43 & 5 & 0.25 & $2.00(0.45-8.96)$ & 0.14 \\
\hline G/G & 7 & 0.50 & 5 & 0.25 & $0.24(0.09-0.68)$ & 0.007 \\
\hline A allele & 8 & 0.29 & 25 & 0.63 & $4.17(1.47-11.80)$ & 0.007 \\
\hline G allele & 20 & 0.71 & 15 & 0.38 & & \\
\hline
\end{tabular}

In GD patients a significantly decreased frequency of A/A genotype $(p<0.05)$ in comparison with controls was observed. Statistically significant differences $(p<0.05)$ in frequencies of A and G alleles between the studied groups were found and $O R$ analysis confirmed the relationship between $G D$ risk and presence of the $G$ allele in the $A 49 G$ polymorphic site. Statistical analysis results are shown in Table III.

The results of analysis of CTLA-4 CT60 (A/G) polymorphism distribution in AITD (HT and GD) patients in comparison with the control group were statistically significant $(p<0.05)$, regarding $A$ and $G$ allele frequencies, with higher $A$ allele frequency in the AITD group. The distribution of genotypes and OR values were as follows: CTLA-4 CT60 A/A: 0.69 AITD vs. 0.50 control group, $\mathrm{OR}=2.23,95 \% \mathrm{Cl}$ : $0.75-6.66, p=0.15 ;$ CTLA-4 CT60 A/G: 0.26 AITD vs. 0.30 control group, $\mathrm{OR}=0.83,95 \% \mathrm{Cl}$ : 0.26-2.69, $p=0.75$; CTLA-4 CT60 G/G: 0.05 AITD vs. 0.20 control group, $\mathrm{OR}=0.20,95 \% \mathrm{Cl}: 0.03-1.20, p=0.08$. The frequency and OR value for A allele: 0.82 AITD vs. 0.65 control group, $\mathrm{OR}=2.48,95 \% \mathrm{Cl}$ : $1.05-5.83$, $p=0.04$ and for $\mathrm{G}$ allele: 0.18 AITD vs. 0.35 control group, $\mathrm{OR}=0.40,95 \% \mathrm{Cl}$ : 0.17-1.12.

The CTLA-4 CT60 haplotypes were also assessed in HT and GD patients separately, and compared with the control group. We did not observe statistically significant differences in frequencies of $A / A$, $\mathrm{A} / \mathrm{G}, \mathrm{G} / \mathrm{G}$ genotypes or allele ( $\mathrm{A}$ and $\mathrm{G}$ ) distribution between $\mathrm{HT}$ patients and the control group $(p>0.05)$.
In the GD group, we also did not observe statistically significant differences in CTLA-4 CT60 genotypes (A/A, A/G, G/G) or allele ( $A$ and $G$ ) distribution in comparison with the control group $(p>0.05)$.

Concerning the distribution of CTLA-4 1822 genotypes (C/C, C/T, T/T) and allele ( $\mathrm{C}$ and $\mathrm{T}$ ) frequencies, we did not find statistically significant differences between the AITD (HT and GD) and control group $(p>0.05)$.

The frequencies of CTLA-4 1822 haplotypes estimated separately for HT and GD patients - were not statistically significant as compared with the control group $(p>0.05)$.

\section{Discussion}

Many studies focusing on the genetic background of AITD have confirmed that two common forms of autoimmune thyroid disorder, i.e., GD and $H T$, are very heterogenic with complex etiopathogenesis involving genetic and environmental interactions. It was postulated that cytotoxic T lymphocyte antigen 4 (CTLA-4) encoded by the CTLA-4 gene plays a fundamental role in susceptibility to AITD $[3,8,16,17,19]$.

The present study confirmed that CTLA-4 A49G genotype might increase the susceptibility to AITD development. Especially, the frequency of A/G genotype was significantly increased in the AITD group as well as the frequency of the $G$ allele, whereas A/A genotype seemed to have a protective effect. These results are in accordance with popula- 
tion-based studies in Europe and Asia [21, 22]. Although A49G dimorphism frequency does not change significantly in relation to age or gender, it is different in various ethnic groups: the A49 allele predominates in whites, whereas the G49 allele is most common in the Asian population [22]. In the latter, patients with $A / G$ heterozygosity more frequently develop HT and GD, although large heterogeneity is noticeable [21, 22]. The present study showed that the G49 allele was statistically more frequent in AITD patients, which resembles rather Asian population studies. However, it should be stressed that the population-based studies were not ethnically homogeneous and an association of the G49 allele with the disease is observed in both European and Asian populations.

The results of the present study indicated that CTLA-4 A/G genotype might influence the development of HT in Polish patients. A strong association of A/G genotype with HT was documented. Moreover, the $G$ allele was more frequent in HT cases. These findings are in agreement with the observations of many studies focused on CTLA-4 A49G polymorphism in HT patients [13, 22, 23]. However, the results published by Kucharska et al. [24] showed significantly increased frequency of $G / G$ genotypes in Polish patients with HT.

Regarding the other studied form of AITD, i.e., GD, it should be pointed out that the CTLA-4 gene is recognized as the most important genetic factor in GD development. Moreover, G/G genotype in CTLA-4 A49G polymorphism is suggested to be an important risk factor of thyroid-associated orbitopathy (TAO) [25]. In the present study an association of $\mathrm{G} / \mathrm{G}$ genotype with ophthalmopathy was not observed. Similar results were obtained by Bednarczuk et al. [16] involving, among others, a Polish population.

Regarding CTLA-4 A49G polymorphism in GD patients, the present study indicated significantly increased $G$ allele frequency and significantly decreased frequency of A/A genotype. Additionally, OR analysis suggested that the increased frequencies of $A / G$ and $G / G$ genotypes might influence the susceptibility to GD. Similarly to these results, the significant association of $A 49 G$ polymorphism with GD has been confirmed by others [16, 19], particularly higher frequencies of the $G$ allele and $G / G$ genotype and lower frequency of A/A genotype in GD as compared with HT patients [16, 22, 26].

However, some published studies have found no linkage between the CTLA-4 locus and GD in UK and Japanese populations [27, 28]. It is also suggested that CTLA-4 polymorphisms do not determine the total phenotype of autoimmune thyroid diseases [29]. Such controversial results and the fact that some individuals bearing $\mathrm{G} / \mathrm{G}$ genotype of CTLA-4 A49G polymorphism do not develop autoimmune disease whereas others with A/A genotype suffer from AITD are postulated to be connected with the multifactorial etiology of HT and GD, including a close association with MHC class II alleles [12].

There was no positive correlation between significantly increased Tg-Ab and TSH values and presence of the $\mathrm{G}$ allele in CTLA-4 A49G polymorphism in AITD patients included in the present study. However, others have found an association between the G49 allele and higher levels of thyroid antibodies, including Tg-Ab and TPO-Ab as well as high TSH value $[23,29,30]$.

Regarding CTLA-4 CT60 polymorphism, no statistically significant differences were found in frequencies of genotypes (A/A, AG, G/G) and alleles between HT and/or GD patients and controls, although weak differences concerning the A allele and A/A genotype between the studied groups were observed and OR analysis suggested that an increased A allele frequency as well as A/A genotype might influence the risk for AITD (GD and/or $\mathrm{HT}$ ). These results, however, did not confirm the observations of other authors referring to increased frequency of the $\mathrm{G}$ allele in CTLA-4 CT60 polymorphism connected with the susceptibility to AITD, particularly GD [15, 17, 19, 31-33]. Additionally, Petrone et al. [15] found significantly increased G allele frequency in GD patients with TAO. In the present study such an association in the GD group was not observed, but the number of patients with ophthalmopathy was very small.

An interesting observation of frequent co-occurrence of the $\mathrm{G}$ allele in CTLA-4 CT60 and the $\mathrm{G}$ allele in CTLA-4 A49G polymorphisms in GD patients from a Taiwanese population was reported [33]. In the present study such a relationship was not observed.

The study did not reveal significantly higher T allele frequency in CTLA-4 1822 C/T polymorphism, which has been recognized as predisposing to GD in different populations [34]. Moreover, an association of the T allele with TAO was found [34], but it was not confirmed in GD patients included in the present study. However, the obtained results of OR analysis suggested a possible association between $\mathrm{C} / \mathrm{C}$ genotype and the risk of AITD development (GD and/or HT).

The obtained results concerning CT 60 and 1822 C/T SNPs, dissimilar to other studies, are probably associated with the small number of cases.

However, the importance of the present study should be stressed. Primarily, it is one of the very few studies involving Polish patients and can be regarded as an initial population-based study. Moreover, the association of CTLA-4 CT60 as well as $1822 \mathrm{C} / \mathrm{T}$ polymorphisms with HT and GD has not been widely studied so far, not only in the Polish population.

In conclusion, several reasons for difficulties affecting analysis of functional polymorphism in a population and in individual patients should be stressed. First of all, interactions between many 
SNPs in the human genome as well as individual interactions between different polymorphisms (e.g., coexistence of A49G and 1822 C/T SNPs) within coding and noncoding regions of the genes are common. Additionally, a complex mechanism of autoimmune diseases involving different genes (immune regulatory genes, thyroid-specific genes) is documented. Recently, gene-gene interactions (mainly between HLA-DR and CTLA-4) and heterogeneous population effects have been shown to constitute a key mechanism in the etiology of both $\mathrm{GD}$ and $\mathrm{HT}[3,15]$. Finally, the differences in the assessment of relative risks for AITD development are caused by the genetic background of the population, many affected individuals, gene penetration as well as by use of a specific logistic regression approach.

\section{References}

1. Wang C, Crapo LM. The epidemiology of thyroid disease and implications for screening. Endocrinol Metab Clin North Am 1997; 26: 189-218.

2. Hemminki K, Li X, Sundquist J, Sundquist K. The epidemiology of Graves' disease: evidence of a genetic and an environmental contribution. J Autoimmun 2010; 34: J307-13.

3. Tomer Y. Genetic susceptibility to autoimmune thyroid disease: past, present, and future. Thyroid 2010; 20: 715-25.

4. Tomer Y, Huber A. The etiology of autoimmune thyroid disease: a story of genes and environment. J Autoimmun 2009; 32: 231-9.

5. Vieland VJ, Huang Y, Bartlett C, Davies TF, Tomer Y. A multilocus model of the genetic architecture of autoimmune thyroid disorder, with clinical implications. Am J Hum Genet 2008; 82: 1349-56.

6. Hodge SE, Ban Y, Strug LJ, et al. Possible interaction between HLA-DRbeta1 and thyroglobulin variants in Graves' disease. Thyroid 2006; 16: 351-5.

7. Tomer Y, Davies TF. Searching for the autoimmune thyroid disease susceptibility genes: from gene mapping to gene function. Endocr Rev 2003; 24: 694-717.

8. Tomer Y, Ban Y, Concepcion E, et al. Common and unique susceptibility loci in Graves and Hashimoto diseases: results of whole-genome screening in a data set of 102 multiplex families. Am J Hum Genet 2003; 73: 736-47.

9. Brunner MC, Chambers CA, Chan FK, Hanke J, Winoto A, Allison JP. CTLA-4-Mediated inhibition of early events of T cell proliferation. J Immunol 1999; 162: 5813-20.

10. Manzotti CN, Tipping H, Perry LC, et al. Inhibition of human T cell proliferation by CTLA- 4 utilizes CD80 and requires CD25+ regulatory T cells. Eur J Immunol 2002; 32: 2888-96.

11. Mäurer M, Loserth S, Kolb-Mäurer A, et al. A polymorphism in the human cytotoxic T-lymphocyte antigen 4 (CTLA4) gene (exon $1+49)$ alters T-cell activation. Immunogenetics 2002; 54: 1-8.

12. Kouki T, Sawai Y, Gardine CA, Fisfalen ME, Alegre ML, DeGroot LJ. CTLA-4 gene polymorphism at position 49 in exon 1 reduces the inhibitory function of CTLA-4 and contributes to the pathogenesis of Graves' disease. J Immunol 2000; 165: 6606-11.

13. Yeșilkaya E, Koç A, Bideci A, et al. CTLA4 gene polymorphisms in children and adolescents with autoimmune thyroid diseases. Genet Test 2008; 12: 461-4.
14. Kavvoura FK, Akamizu T, Awata T, et al. Cytotoxic T-lymphocyte associated antigen 4 gene polymorphisms and autoimmune thyroid disease: a meta-analysis. J Clin Endocrinol Metab 2007; 92: 3162-70.

15. Petrone A, Giorgi G, Galgani A, et al. CT60 single nucleotide polymorphisms of the cytotoxic T-lymphocyte-associated antigen-4 gene region is associated with Graves' disease in an Italian population. Thyroid 2005; 15: 232-8.

16. Bednarczuk T, Hiromatsu Y, Fukutani T, et al. Association of cytotoxic T-lymphocyte-associated antigen-4 (CTLA-4) gene polymorphism and non-genetic factors with Graves' ophthalmopathy in European and Japanese populations. Eur J Endocrinol 2003; 148: 13-8.

17. Ueda $\mathrm{H}$, Howson JM, Esposito L, et al. Association of the T-cell regulatory gene CTLA4 with susceptibility to autoimmune disease. Nature 2003; 423: 506-11.

18. Takahashi M, Kimura A. HLA and CTLA4 polymorphisms may confer a synergistic risk in the susceptibility to Graves' disease. J Hum Genet 2010; 55: 323-6.

19. Bicek A, Zaletel K, Gaberscek S, et al. 49A/G and CT60 polymorphisms of the cytotoxic T-lymphocyteassociated antigen 4 gene associated with autoimmune thyroid disease. Hum Immunol 2009; 70: 820-4.

20. Johnson GC, Esposito L, Barratt BJ, et al. Haplotype tagging for the identification of common disease genes. Nat Genet 2001; 29: 233-7.

21. Chistiakov DA, Turakulov RI. CTLA-4 and its role in autoimmune thyroid disease. J Mol Endocrinol 2003; 31: 21-36.

22. Kavvoura FK, Akamizu T, Awata T, et al. Cytotoxic T-lymphocyte associated antigen 4 gene polymorphisms and autoimmune thyroid disease: a meta-analysis. J Clin Endocrinol Metab 2007; 92: 3162-70.

23. Terauchi $M$, Yanagawa $T$, Ishikawa $N$, et al. Interactions of HLA-DRB4 and CTLA-4 genes influence thyroid function in Hashimoto's thyroiditis in Japanese population. J Endocrinol Invest 2003; 26: 1208-12.

24. Kucharska A, Wiśniewska A, Rymkiewicz-Kluczyńska B. The frequency of CTLA-4 gene polymorphism at position 49 exon 1 in children with Hashimoto's thyroiditis. Endokrynol Diabetol Chor Przemiany Materii Wieku Rozw 2006; 12: 163-6.

25. Vaidya B, Imrie H, Perros P, et al. Cytotoxic T lymphocyte antigen-4 (CTLA-4) gene polymorphism confers susceptibility to thyroid associated orbitopathy. Lancet 1999; 354: 743-4.

26. Kouki T, Gardine CA, Yanagawa T, Degroot LJ. Relation of three polymorphisms of the CTLA-4 gene in patients with Graves' disease. J Endocrinol Invest 2002; 25: 208-13.

27. Nithiyananthan R, Heward JM, Allahabadia A, Franklyn JA, Gough SC. Polymorphism of the CTLA-4 gene is associated with autoimmune hypothyroidism in the United Kingdom. Thyroid 2002; 12: 3-6.

28. Tomoyose T, Komiya I, Takara M, et al. Cytotoxic T-lymphocyte antigen-4 gene polymorphisms and human T-cell lymphotrophic virus-1 infection: their associations with Hashimoto's thyroiditis in Japanese patients. Thyroid 2002; 12: 673-7.

29. Tomer Y, Greenberg DA, Barbesino G, Concepcion E, Davies TF. CTLA-4 and not CD28 is a susceptibility gene for thyroid autoantibody production. J Clin Endocrinol Metab 2001; 86: 1687-93.

30. Zaletel K, Krhin B, Gaberscek S, Pirnat E, Hojker S. The influence of the exon 1 polymorphism of the cytotoxic $T$ lymphocyte antigen 4 gene on thyroid antibody production in patients with newly diagnosed Graves' disease. Thyroid 2002; 12: 373-6. 
31. Mayans S, Lackovic K, Nyholm C, et al. CT60 genotype does not affect CTLA-4 isoform expression despite association to T1D and AITD in northern Sweden. BMC Med Genet 2007; 8: 3.

32. Ban Y, Tozaki T, Taniyama M, Tomita M. Association of a CTLA-4 3' untranslated region (CT60) single nucleotide polymorphism with autoimmune thyroid disease in the Japanese population. Autoimmunity 2005; 38: 151-3.

33. Weng YC, Wu MJ, Lin WS. CT60 single nucleotide polymorphism of the CTLA-4 gene is associated with susceptibility to Graves' disease in the Taiwanese population. Ann Clin Lab Sci 2005; 35: 259-64.

34. Vaidya B, Oakes EJ, Imrie H, et al. CTLA4 gene and Graves' disease: association of Graves' disease with the CTLA4 exon 1 and intron 1 polymorphisms, but not with the promoter polymorphism. Clin Endocrinol (Oxf) 2003; 58: 732-5. 